\title{
EINFLUSS VON ENTWÄSSERUNG UND LANDWIRTSCHAFT- LICHER NUTZUNG AUF DIE DURCHLÄSSIGKEIT DES MOORBODENS
}

\author{
Rudolf EgGelsmann
}

\author{
Staatl. Moor-Versuchsstation in Bremen, Direktor: Prof. Dr. W. Baden \\ und \\ TIMO MÄKELÄ
}

Finnischer Moorkulturverein, Leteensuo Moorversuchsstation

Eingegangen am 17. 2. 1964

\begin{abstract}
Zwischen der finnischen und deutschen Moorforschung und Moorkunde haben von Anfang an besonders enge Beziehungen bestanden. Mögen auch zwischen den deutschen und finnischen Moorgebieten vor allem bemerkenswerte klimatische Unterschiede vorherrschen, so gelten dennoch für beide moorreichen Länder manch grundsätzliche Erkenntnisse bei einer rationellen Moornutzung gleichermassen. So ist auch das mit der nachstehenden Gemeinschaftsarbeit aufgegriffene Problem hier wie dort gleich bedeutungsvoll.

Als korrespondierendes Mitglied des Finnischen Moorvereins weiss ich den regen Gedankenaustausch der finnischen und deutschen Moorfachleute besonders hoch zu schätzen und begrüsse deshalb die nachstehende Gemeinschaftsarbeit ausserordentlich.
\end{abstract}

\author{
Prof. W. B a d e n \\ Direktor \\ der Staatl. Moorversuchsstation
}

\section{Einleitung}

Die Durchlässigkeit des Moorbodens und deren Beeinflussung durch Entwässerung und Kultivierung finden in den letzten Jahren nicht nur in Deutschland, sondern ebenso sehr in Finnland reges Interesse. BADEN \& EGGELSmann (3) haben neben manchen anderen deutschen Autoren kürzlich ihre Ergebnisse von über sechsjährigen Erfahrungen zusammengefasst und die bis dahin vorliegende Literatur diskutiert. Von forstlicher Seite haben in Finnland Huikari (8) und Sarasto $(13,14)$ der Durchlässigkeit der Torfe ihre Aufmerksamkeit gewidmet, auch HelE- 
NELund (7) berührte dieses Problem. Im August 1963 ergab sich in der Moorversuchsstation Leteensuo Gelegenheit dieser Frage auch dort nachzugehen. Im Anschluss daran kam es zu gemeinsamen Untersuchungen mit ersten, auch unter finnischen Verhältnissen bemerkenswerten Ergebnissen.

\section{Örtliche Verhältnisse}

Die Moorversuchsstation Leteensuo liegt am Westrand des Leteensuo-Moores, eines typischen südfinnischen Hochmoores, etwa $100 \mathrm{~km}$ nördlich der finnischen Südküste $\left(24^{\circ} 15^{\prime} \mathrm{E} ; 61^{\circ} 04^{\prime} \mathrm{N}\right)$ zwischen 80 und $83 \mathrm{~m}$ über dem Meere, nahe Hämeenlinna zwischen zwei Binnenseen. Der Untergrund des Moores besteht aus schwerem Ton.

Die mittlere Jahrestemperatur $(1931 / 60)$ beträgt $3.9^{\circ} \mathrm{C}$ (Februar $-8.0^{\circ} \mathrm{C}$ und Juli $16.9^{\circ} \mathrm{C}$ ). Der jährliche Niederschlag ist $514 \mathrm{~mm}$, davon fallen im Sommerhalbjahr (Mai-September) $290 \mathrm{~mm}$. Eine dauernde, Mitte März etwa $40 \mathrm{~cm}$ dicke Schneedecke liegt durchschnittlich vom 5. Dezember bis 20. April. Die Vegetationszeit (Tagesmittel über $+5^{\circ} \mathrm{C}$ ) dauert rund 160 Tage.

Das Moorgebiet umfasst insgesamt etwa 400 ha; das grössere, mittlere Areal ist noch meist unkultiviertes Hochmoor; die niedermoorartigen Randgebiete sind dagegen überwiegend zur landwirtschaftlichen Nutzung als Grünland und Acker hergerichtet. Rindell, Lindberg und Malm $(12,10,11)$ haben über die Geologie, die natürliche Vegetation sowie die Höhenverhältnisse und die Moorstratigraphie von Leteensuo berichtet. Vom letzteren stammt auch der Plan der Entwässerung, Kultivierung und Einrichtung der Moor-Versuchswirtschaft im Jahre 1908. Einige Teilflächen sind nach Art und Menge in verschiedener Weise unter Zuhilfenahme von Mineralboden kultiviert worden.

\section{Zweck und Art der Untersuchungen}

Die Versuchsflächen, auf denen wir unsere Untersuchungen anstellten, sind in beträchlichen Zeitabständen entwässert und kultiviert (Abb. 1 und Tab. 1). Gerade

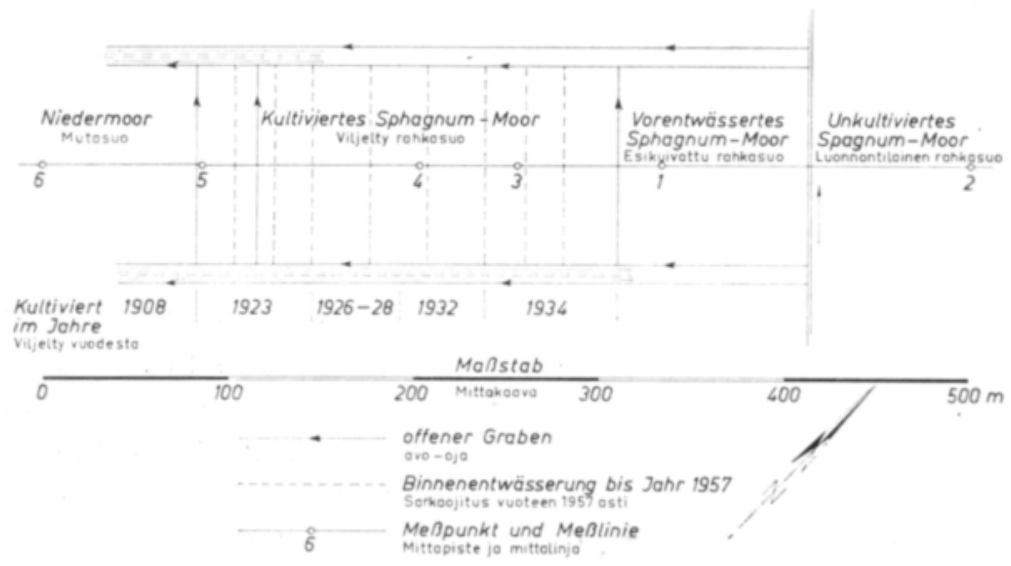

Abb. 1. Lageskizze der Versuchsflächen in Leteensuo mit den Messpunkten 1 bis 6. 
diese Tatsache gab den Anstoss, innerhalb des Sphagnum-Moores der Frage der bodenphysikalischen Wandlungen in den Torfen in Abhängigkeit von der Zeit nachzugehen.

Tab. 1. Ubersicht über Entwässerung, Kultivierung, landwirtschaftliche Nutzung und pH-Wert das Moores Leteensuo.

\begin{tabular}{|c|c|c|c|c|c|c|c|}
\hline \multirow{2}{*}{$\begin{array}{l}\text { Mess- } \\
\text { punkt }\end{array}$} & \multirow{2}{*}{$\begin{array}{c}\text { Vorent- } \\
\text { wässert } \\
\text { seit dem } \\
\text { Jahre }\end{array}$} & \multirow{2}{*}{$\begin{array}{l}\text { Ent- } \\
\text { wässert } \\
\text { seit dem } \\
\text { Jahre }\end{array}$} & \multirow{2}{*}{$\begin{array}{l}\text { Kultiviert } \\
\text { im Jahre }\end{array}$} & \multicolumn{2}{|c|}{ Mineralbodenzugabe } & \multirow{2}{*}{$\begin{array}{l}\text { Nutzungsart } \\
\text { und - zeit (Jahre) } \\
\text { Grünland Acker }\end{array}$} & \multirow{2}{*}{$\begin{array}{c}\text { pH-Wert } \\
\left(\mathrm{H}_{2} \mathrm{O}\right) \\
\text { in der } \\
\text { Oberkrume }\end{array}$} \\
\hline & & & & $\mathrm{m}^{3} / \mathrm{ha}$ & Art & & \\
\hline
\end{tabular}

\begin{tabular}{rrrrcrrrr}
\hline 2 & nicht entwässert & - & - & - & - & - & 3.7 \\
1 & 1903 & 1961 & - & - & - & - & - & 3.8 \\
3 & 1903 & 1932 & 1934 & 300 & Ton/Savea & 20 & 9 & 5.6 \\
4 & 1903 & 1930 & 1932 & 400 & Sand/Hiekkaa & 19 & 12 & 5.9 \\
5 & 1903 & 1921 & 1923 & 300 & Ton/Savea & 21 & 19 & 5.7 \\
6 & 1903 & 1908 & 1908 & & & & &
\end{tabular}
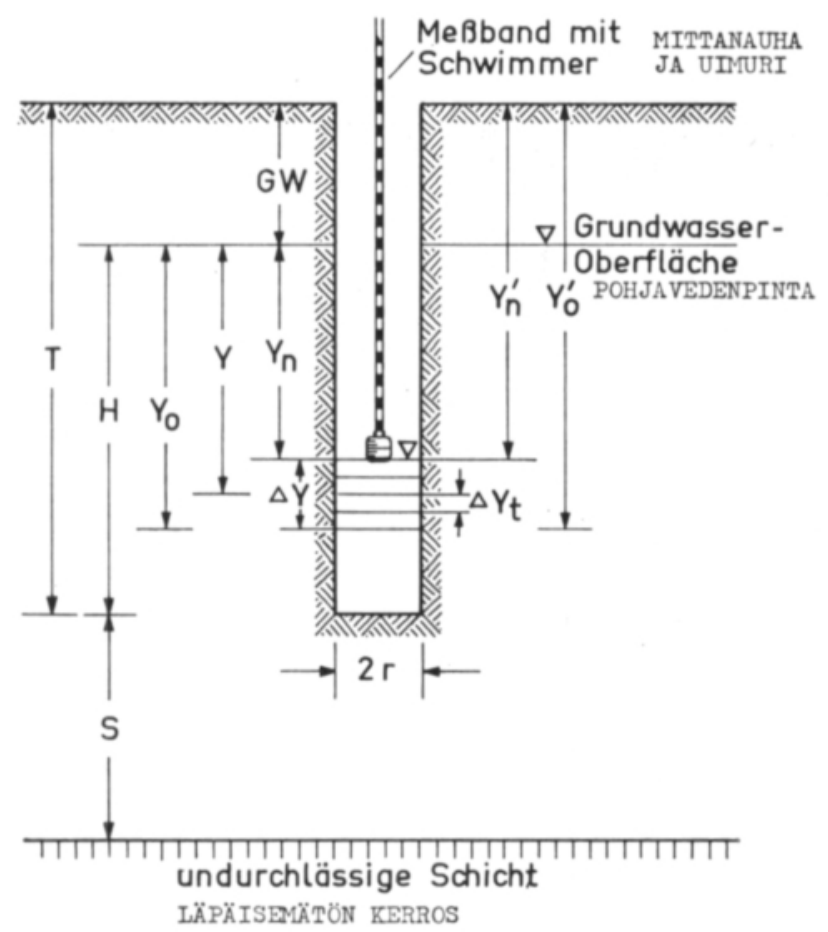

Abb. 2. Schema der Bohrlochmethode zum Bestimmen der Bodendurchlässigkeit nach HoogноuDT in Anlehnung an van BEERS

a) Neben einer erneuten Höhenaufnahme zum Vergleich mit der von MaLM aus dem Jahre 1903 sind von uns an den Messpunkten $1-5$ folgende weitere Untersuchungen angestellt: 
b) Messung des Grundwasserstandes unter der Geländeoberfläche;

c) Bestimmung der Bodendurchlässigkeit im Felde in den Bodentiefen von 60 bis $100 \mathrm{~cm}$ aus der Steiggeschwindigkeit des Grundwassers in Erdbohrlöchern bekannter Weite und Tiefe nach HooghoudT-ERNST, beschrieben durch van BEERS, (4) (Abb. 2).

d) Entnahme von Bodenproben aus den gleichen Bodentiefen (wie c) zur bodenphysikalischen Analyse in Leteensuo und Bremen ${ }^{1}$ );

e) Ermittlung des pH-Wertes im Oberboden in Leteensuo.

\section{Ergebnisse und ihre Diskussion}

a) Höhenverlust. Die im Profilschnitt (Abb. 3) vergleichend dargestellten Höhenaufnahmen lassen von dem Messpunkt 1 bis 6 heute ein beträchtliches Oberflächengefälle erkennen, welches 1903 längst nicht so ausgeprägt vorhanden war. Die sich daraus ergebenden Oberflächensenkungen sind zahlenmässig mit den zugehörenden

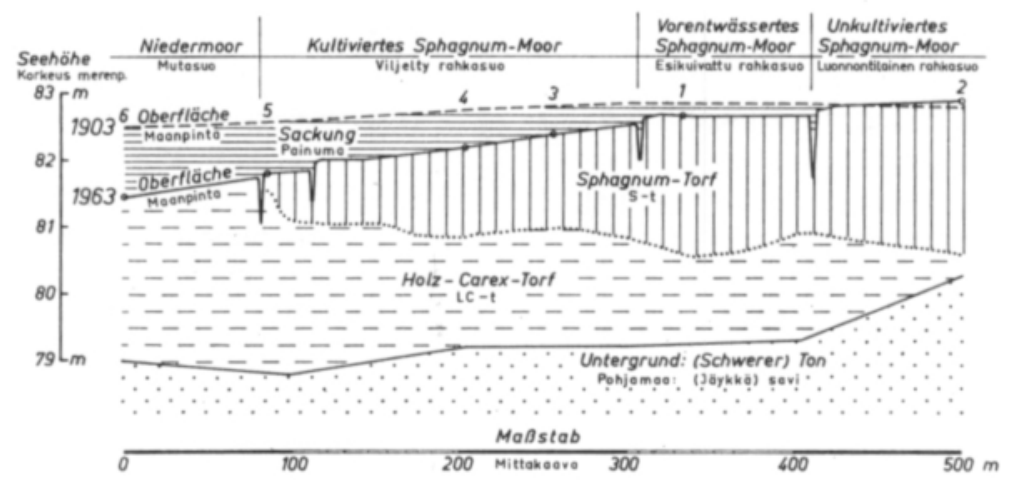

Abb. 3. Profilschnitt durch die Messpunkte 1 bis 6 in Leteensuo.

Tab. 2. Ergebnisse der Geländeuntersuchungen.

\begin{tabular}{|c|c|c|c|c|c|c|c|c|}
\hline \multirow{2}{*}{$\begin{array}{l}\text { Mess- } \\
\text { punkt }\end{array}$} & \multicolumn{2}{|c|}{$\begin{array}{c}\text { Entwässerungszeit } \\
\text { Jahre }\end{array}$} & \multicolumn{3}{|c|}{ Oberflächensenkung in $\mathrm{m}$} & \multirow{2}{*}{$\begin{array}{l}\text { Grund- } \\
\text { wasser } \\
\text { unter } \\
\text { Flur m }\end{array}$} & \multicolumn{2}{|c|}{ Bodendurchlässigkeit } \\
\hline & $\underset{\text { Vorentw. }}{\text { Jahr }}$ & $\begin{array}{l}\text { binnen } \\
\text { entw. }\end{array}$ & gesamt & $\begin{array}{l}\text { davon } \\
\text { Sackung }\end{array}$ & $\begin{array}{c}\text { Torf- } \\
\text { verzehr }\end{array}$ & & $\mathrm{m} / \mathrm{Tag}$ & Charakteristik \\
\hline 2 & - & - & $(+0.12)$ & - & - & 0.25 & 0.84 & stark/ \\
\hline 1 & 57 & 2 & 0.20 & 0.20 & - & 0.35 & 0.65 & mässig/ \\
\hline 3 & 29 & 31 & 0.45 & 0.38 & 0.07 & 0.44 & 0.04 & sehr schwach/ \\
\hline 4 & 27 & 33 & 0.57 & 0.47 & 0.10 & 0.71 & 0.03 & sehr schwach/ \\
\hline 5 & 18 & 42 & 0.77 & 0.62 & 0.15 & 0.79 & 0.18 & gering/ \\
\hline 6 & 5 & 55 & 1.05 & & & & & \\
\hline
\end{tabular}

1) Für freundliche Unterstützung danken wir den Herren Reg.-Chemierat Dr. $S$ t e in $f$ a $t$ und Dr. Grosse-Bra u c man n an der Staatl. Moor-Versuchsstation in Bremen. 
Entwässerungszeiten in Tab. 2 aufgeführt. Dabei ist zu beachten, dass die Höhendifferenzen zwar genau (in $\mathrm{cm}$ ) angegeben sind, jedoch mit einem Fehler bis zu $\pm 10 \mathrm{~cm}$ behaftet sein können; denn die Lage der Höhenpunkte des Jahres 1903 konnten naturgemäss 1963 nicht exakt rekonstruiert werden. Trotzdem ist ersichtlich, dass mit zunehmender Entwässerungszeit die Oberflächensenkungen - als Folge der durch Entwässerung und Nutzung verursachten Volumenänderungen im Torf - grösser werden.

Bei den fogenden Vergleichen über das Sphagnum-Moor werden die Messpunkte 5 und 6 ausser acht gelassen, da sie im Niedermoor liegen (Abb. 3).

Nach den in Tab. 1 genannten Angaben der Nutzungsart und -zeit sowie der derzeitigen Bodenazidität konnte nach einem Vorschlag von EGGELSMANN (5) der während der Zeit der Ackernutzung eingetretene Torfverzehr geschätzt werden (Tab. 2). Danach war es weiter möglich für den Sphagnumtorf als Differenz die eigentliche Sackung infolge der Entwässerung zu überschlagen. Die sich andeutungsweise ergebende Sackung-Zeit-Kurve (Abb. 4 oben) ist recht ähnlich der aus der

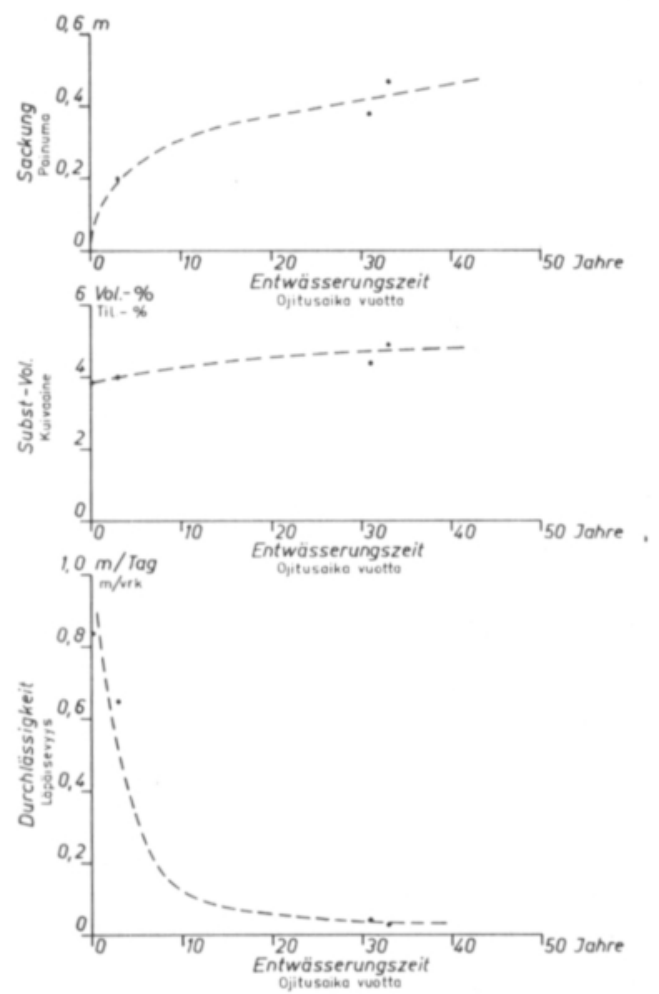

Abb. 4. Vermutlicher zeitlicher Verlauf von Sackung, Substanzvolumen und Durchlässigkeit.

Moor-Versuchswirtschaft Königsmoor bei Hamburg (5) und der aus anderen Mooren $(9,7)$. Die Sackung im Sphagnum-Moor der Versuchsstation Leteensuo dürfte auch heute noch nicht völlig beendet sein.

b) Grundwasser. Das Grundwasser stand am 13. August 1963 bei Messpunkt 2, 1 und 3 verhältnismässig flach an, dagegen an den Punkten 4 und 5 erheblich 
tiefer (Tab. 2). Diese sporadischen Messergebnisse lassen jedoch noch keine verlässliche Deutung zu, sollten vielmehr zu weiteren und regelmässigen Grundwasserbeobachtungen anregen, wie sie inzwischen eingeleitet waren.

c) Durchlässigkeit. Die Durchlässigkeit (Tab. 2) im schwach zersetzten Sphagnumtorf nimmt anfangs wenig (von Messpunkt 2 nach 1) dann sehr stark ab (von Messpunkt 1 nach 3 und 4). Dieser zeitliche Verlauf der Durchlässigkeit ist graphisch in Abb. 4 (unten) angedeutet, er ist ein Analogon zum Sackungsverlauf. Eine ähnliche Durchlässigkeitsabnahme ist für manche andere europäische Moore unter Beweis gestellt (3), ist auch in einem kanadischen Moor beobachtet (6) und nunmehr auch für das Sphagnum-Moor der Versuchsstation Leteensuo belegt.

Tab. 3 Ergebnisse der Bodenanalysen

\begin{tabular}{|c|c|c|c|c|c|c|c|c|}
\hline $\begin{array}{c}\text { Probe } \\
\text { von } \\
\text { Mess- } \\
\text { punkt }\end{array}$ & Torfart & $\begin{array}{c}\text { Vertor- } \\
\text { fungs- } \\
\text { grad } \\
\text { (n. Kep- } \\
\text { peler) \% }\end{array}$ & $\begin{array}{l}\text { Ver- } \\
\text { brennl. } \\
\text { Stoffe } \\
\text { Gew.- } \\
\% \text { tr. }\end{array}$ & $\begin{array}{l}\text { Mineral.- } \\
\text { Stoffe } \\
\text { Gew.- } \\
\% \mathrm{tr} .\end{array}$ & $\begin{array}{l}\text { spez. } \\
\text { Gewicht } \\
\mathrm{g} / \mathrm{cm}^{3}\end{array}$ & $\begin{array}{l}\text { Volumen- } \\
\text { Gewicht } \\
\text { tr. } \\
\mathrm{g} / \mathrm{cm}^{3}\end{array}$ & $\begin{array}{c}\text { Substanz- } \\
\text { Volumen } \\
\%\end{array}$ & $\begin{array}{l}\text { Poren- } \\
\text { ziffer } \\
\varepsilon\end{array}$ \\
\hline 2 & $\begin{array}{c}\text { Sphagnum-fuscum- } \\
\text { Torf }\end{array}$ & 9.8 & 96.90 & 3.10 & 1.65 & 0.064 & 3.9 & 24.0 \\
\hline 1 & -- & 10.7 & 98.85 & 1.15 & 1.62 & 0.065 & 4.0 & 24.7 \\
\hline 3 & -- & 12.5 & 98.75 & 1.25 & 1.61 & 0.070 & 4.4 & 22.0 \\
\hline 4 & -- & 27.1 & 98.42 & 1.58 & 1.56 & 0.076 & 4.9 & 19.4 \\
\hline 5 & $\begin{array}{c}\text { Carex-Sphagnum- } \\
\text { Torf }\end{array}$ & 66.7 & 88.14 & 11.86 & 1.63 & 0.150 & 9.2 & 9.9 \\
\hline
\end{tabular}

d) Bodenphysikalische Analysen. Vergleicht man damit nun die Ergebnisse der bodenphysikalischen Analysen (Tab. 3), so ist zu bemerken, dass mit zunehmender Entwässerungszeit sowohl Vertorfungsgrad als auch Mineralstoffgehalt, Volumengewicht (trocken) und Substanzvolumen zugenommen haben, letzteres ist in Abb. 4 (Mitte) skizziert. Diese Änderungen lassen darauf schliessen, dass sich die Torfe durch den Wasserentzug nicht nur mechanisch dichter gelagert haben (Verringerung des Porenvolumens und damit der Porenziffer $\varepsilon=$ Porenvolumen : Substanzvolumen), sondern dass die damit zeitweilig einhergehende Belüftung auch chemisch (Oxydation) und biologisch auf die Torfsubstanz eingewirkt hat. Die starke Abnahme der Durchlässigkeit lässt weiter darauf schliessen, dass nicht nur der gesamte Porenraum geringer geworden ist, sondern dass sich in viel stärkerem Umfang der Anteil an Makroporen im Boden verringert und sich dafür der an Mittel- oder Mikroporen vermehrt hat. Da es z.Zt. nicht möglich ist, zuverlässig die pF-Werte in Torfen zu bestimmen, so kann dieser Hypothese experimentell noch nicht nachgegangen werden; entsprechende Versuche sind in Bremen in Gange.

Die von Zunker (zit. Wechmann, 16), Helenelund (7) und Sarasto (13) experimentell an Torf- und Muddeproben mit zunehmender Belastung ermittelte Durchlässigkeitsabnahme, ist kulturtechnisch dem Einfluss der Sackung auf die Durchlässigkeit gleichzusetzen. 


\section{Schlussfolgerungen}

Das in der Praxis der Moorkultur wiederholt beobachtete Phänomen, dass anfänglich ausreichend bemessene Drän- und Grabenabstände sich nach $2-3$ Jahrzehnten - besonders in nassen Jahren - als zu gering erweisen, haben u.a. BADEN (1) und in den letzten Jahren in Leteensuo auch PEssi ${ }^{1}$ festgestellt und dürfte mit vorstehenden Ergebnissen hinlänglich erklärt sein. Die allgemein in bestimmten Moorkulturtypen (nämlich den $\mathrm{n}$ i ch t tiefgreifend, mechanisch umgestaltenen Moorprofilen) im Laufe der Entwässerung und Nutzung zu erwartende Durchlässigkeitsabnahme, veranlassten Baden \& EgGELsmann (2) hierfür in Nordwesteuropa mehr und mehr die Maulwurfdränung zu empfehlen. Vor allem die schon stärker humifizierten oder auch schon länger entwässerten Torfe mit ihren oft recht geringen Durchlässigkeiten wären mit üblichen Ton- oder Plasticrohrdränen kaum mehr wirtschaftlich zu entwässern. Gerade in diesen Torfen sind für eine Maulwurfdränung besonders gute statische Verhältnisse gegeben, die eine meist ausreichende Lebensdauer unberohrter Maulwurfdräne erwarten lassen (2).

Eine Entwässerung durch ein enges Netz von Gräben ist wegen der Wirtschaftserschwernisse und der kostspieligen Unterhaltung für den Landwirt wohl auch in Finnland aus praktischen und ökonomischen Gründen kaum mehr diskutabel.

In den finnischen Mooren kommt neben der Beseitigung zeitweilig zu hohen Grundwassers vor allem die rasche Abführung des überschüssigen Wassers nach der Schneeschmelze hinzu. Ob und wieweit dies in Mooren möglich ist, ist nicht zuletzt vom Oberflächengefälle abhängig. Erste Beobachtungen in der Frost-Versuchsstation Pelsonsuo an dortigen Maulwurfdränen berechtigen zu weiteren Hoffnungen auch für finnische Moore (Nach mündlicher Mitteilung von Herrn Dr. A. VAlmari).

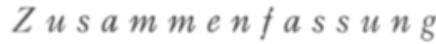

In der südfinnischen Moor-Versuchsstation Leteensuo hat sich - wie auch in anderen Mooren beobachtet - im Sphagnum-Moor mit zunehmender Entwässerungszeit und der dadurch ausgelösten Moorsackung und bodenphysikalischen Wandlungen eine starke Abnahme der Durchlässigkeit ergeben, und dürfte auch dort während nasser Jahre die Ursache unzureichender Bodenentwässerung gewesen sein. Es wird die Frage aufgeworden, ob man dieser Schwierigkeit nicht auch in finnischen Mooren mittels unberohrter Maulwurfdräne Herr werden kann.

1) Nach mündlicher Mitteilung. 


\title{
S C H R I F T U M
}

(1) BADEN, W. 1940. Wird die Wasserführung im Moorboden mit der fortschreitenden Zersetzung Torfe reger oder träger? Mitt. Moorwirtschaft in der Dt. Landeskultur Ztg. 5: 73-75.

(2) Baden, W. \& R. Eggelsmann, 1961. Maulwurfdränung im Moor. Z.f. Kulturtechnik 2: 146 - 166.

(3) - - \& $\quad$ \& 1963. Zur Durchlässigkeit der Moorböden. Z.f. Kulturtechnik u. Flurbereinigung, 4: 226-254.

(4) Beers, W. F. J. van, 1961. Die Bohrlochmethode. Int. Inst. f. Land Reclamation and Improvement. Bull. 1.

(5) Eggelsmann, R. 1960. Über die Höhenänderungen der Mooroberfläche infolge von Sackung und Humusverzehr sowie in Abhängigkeit von Azidität, Atmung und anderen Einflüssen. Mitt. d. Moor-Versuchsst. Bremen 8. Ber. 99-132.

(6) - 1962. Ergänzung der üblichen Moorprofilansprache durch Messung der Durchlässigkeit und der Grundwasserströmungsverhältnisse im freien Felde. - Kolloquium über Fragen des Bodenwasserhaushaltes März 1962 Bochum. Ber. Landesanstalt f. Bodennutzungsschutz, H. 3: $99-106$.

(7) Helenelund, K. V. 1951. On consolidation and settlement of loaded soil-layers. Soil- and Watertechnical Researches, 6, Helsinki (Schwed. m. engl. Ref.).

(8) Huikari, O. 1960. Feldmessungsergebnisse über die Wasserdurchlässigkeit von Torfen. Mitt. Forstl. Forsch.-anst. Finnland 51. 1-26 (Finn. m. dt. Ref.).

(9) KatterA, P. 1954. Om uppskattning av markytans sättning vid torrläggningsarbetena. Nord. Jordbr.forsk. 36 ; $532-537$.

(10) Lindberg, H. 1903. Leteensuon kasvillisuus. S. suovilj. yhd. vuosik.: 264-270.

(11) Malm, E. A. 1903. Tiedonantoja Leteensuon tutkimuksesta Hattulan ja Kalvolan pitäjissä Hämeen lääniä I. Ibid.: $255-263$.

(12) Rindell, A. 1903. Geologisessa mielessä huomattava tulos Leteensuon tutkimisesta. Ibid.: 271-275.

(13) Sarasto, J. 1961. Experiments on the permeability of peat. Suo 12: 24-25 (Finn. m. engl. Ref.).

(14) - 1963. A study on the permeability to water of different kinds of peat. Ibid. 14: $32-$ 36 (Finn. m. engl. Ref.).

(15) Segeberg, H. 1956. Uber den Zusammenhang zwischen den sHumositätsgraden nach von Post, den Vertorfungsgraden nach Keppeler und den spez. Gewichten von Hochmoortorfen. Z.Pfl. Ern. Dgg. u. Bodenkd. 73 (118): 74-85.

(16) Wechmann, A. 1943. Die Durchlässigkeit von Moorböden. Dt. Wasserwirtsch. 38: $136-138$.

S E L O T U S

\section{SUON KUIVATUKSEN JA VILJELYTOIMENPITEIDEN VAIKUTUKSESTA TURPEEN VEDENLÅPÅISYKYKYYN}

\author{
Rudolf EggelsmanN
}

Staatl. Moor-Versuchsstation in Bremen; Direktor: Prof. Dr. W. Baden

TIMO MÄKELÄ

Suoviljelysyhdistys, Leteensuon koeasema

Leteensuon koeaseman rahkasuoviljelyksillä on todettu turpeen vedenläpäisykyvyn huononevan viljelysten vanhetessa. Ojitusajan kasvaessa vedenläpäisykyky heikkenee aluksi vähän, myöhemmin hyvin voimakkaasti (taul. 2; kuv. 4). Samalla on tapahtunut tuntuvaa suonpinnan painumista ja fysikaalisia muutoksia turpeessa (taul. 3). Nämä lienevät pääasialliset syyt viljelysten huonoon kuivatustilaan etenkin märkinä vuosina. Olisi aiheellista kokeilla, voitaisiinko tämä vaikeus myös Suomen olosuhteissa voittaa myyräojituksella. 